\title{
A MULTISCALE APPROACH TO SOLVING ONE DIMENSIONAL INVERSE PROBLEMS*
}

\author{
Eric Miller' Alan Willsky \\ Department of Electrical Engineering and Computer Science \\ Massachusetts Institute of Technology \\ Cambridge, MA. 02139, USA \\ Telephone (617) 253-6172 \\ email: elmiller@athena.mit.edu
}

\begin{abstract}
In this paper we explore a multiresolution approach to solving one dimensional inverse problems. The approach we take is motivated by the work of Chou, Golden, and Willsky [1] and Beylkin, Coifman, and Rokhlin [2]. Specifically, we consider inverse problems described by that class of operators which are made sparse under the action of the wavelet transform [2]. Moreover, statistically-based inversion procedures utilizing multiscale a priori stochastic models are considered [1]. As a concrete example, we examine a deconvolution problem arising in wellbore induction measurement of conductivity.
\end{abstract}

\section{INTRODUCTION}

In this work we consider the utility of applying multiresolution methods to the solution of linear inverse problems. We take this approach above all others specifically because many of the issues arising in the solution of linear inverse problems are exactly those which are handled well by multiresolution methods. First, there exist inverse problems for which the phenomena under consideration may be fractal in nature or characterized in an interesting way by their behavior on a multitude of temporal or spatial scales. Alternatively, the data upon which the inversion is based may exhibit multiscale characteristics as is the case in many sensor fusion problems [1]. Finally, even if neither the phenomena nor the data is inherently multiscale, recent work by Beylkin, Coifman, and Rokhlin

"This work was supported in part by the Office of Naval Research under Grant N00014-91-J-1004, the US Army Resesrch Office under Contract DAAL03-92-G-0115, and the Air Force Office of Scientific Research under Grant AFOSR-92-J-0002.

The work of this author was also supported in part by a fellowship from Schlumberger-Doll Research.
[2] suggests that multiscale methods are a useful tool for purposes of computational efficiency.

We consider inverse problems described by that class of operators which are made sparse under the action of the wavelet transform [2]. Thus, the inversion procedure is carried out in the wavelet transform domain. As with many inverse problems, difficulties associated with ill-posedness and ill-conditioning are overcome via regularization. Because the inversion is performed in scale-space, we employ a statistically-based regularization technique utilizing multiscale a priori models similar to those explored by Chou and Willsky in [1].

The vehicle for our work is an inverse problem arising in the area of geological exploration. Specifically, we are interested in determining the conductivity profile about a wellbore based upon a suite of induction measurements each of which contains conductivity information at different spatial resolutions. This problem is of particular interest because it fulfills all three criteria for the application of multiresolution techniques to inverse problems. That is, conductivity variations are typically fractal in nature, the data conveys information on a variety of scales, and the linear operators describing the measurements process are nearly diagonalized by the wavelet transform.

\section{INDUCTION LOGGING}

Induction logging is a common technique used in geological exploration for determining the conductivity profile of a rock formation surrounding a wellbore. As shown in Figure 1, an induction $\log$ is recorded as a transmitter and receiver coil pair are pulled through the formation. A current in the transmitter induces sympathetic currents in the rock. In turn, these formation currents create a secondary magnetic field resulting in measurable current at the receiver [3]. The problem is to determine the conductivity of the formation given the current signal at the receiver. More 
sophisticated induction logging techniques involve the use of multiple transmitter and receiver pairs all measuring the same rock structure with different pairs sensitive to different areas about the borehole. As the length of each $\log$ becomes long and the number of antenna pairs grows, there exists a problem in terms of performing the inversion in reasonable time and at reasonable cost while still making good use of the vast quantity of available data.

In general, the conductivity in the formation is related in a nonlinear way to the currents observed in the formation. Commonly, the problem is approached by linearizing this relationship about some a priori background conductivity profile [4]. Moreover, it is often assumed that the formation is azimuthally symmetric about the borehole. In this paper, we reduce the problem to the one dimensional case by making the further assumption that the conductivity only varies vertically. In this case, it can be shown [4] that the observations at the $i^{\text {th }}$ receiver, $g_{i}(z)$ are related to the conductivity field, $f(z)$, through the convolution equation

$$
g_{i}(z)=\int T_{i}\left(z-z^{\prime}\right) f\left(z^{\prime}\right) d z^{\prime} \equiv T_{i} f
$$

Where $T_{i}(z)$ is a linearized kernel derived from Maxwell's equations and dependent upon the background conductivity profile. Two examples of the $T_{i}(z)$ from Schlumberger's Array Induction Tool ${ }^{T M}$ are given in Figures 2 demonstrating how information at different resolutions may be available to the inversion routine.

\section{MULTIRESOLUTION PROBLEM STATEMENT}

In [2], Beylkin, Coifman and Rokhlin proved that a large class of operators are made sparse under the action of the wavelet transform and derived a fast algorithm for evaluating integrals containing these kernels. The kernels we consider in conjunction with the induction logging problem are also of the class discussed in [2]. Thus, we are able to exploit the sparse and nearly diagonal structure of these operators in the wavelet domain to construct their inverses using computationally efficient algorithms.

In this work, we adopt the following notation. In one dimension, the scaling function coefficient for a function $f(z)$ corresponding to the basis function at scale $m$ and shift $k, \varphi_{m ; k}(t)$, is given by $f(m ; k)$ while the wavelet coefficient associated with the wavelet $\psi_{m ; k}(t)$ is $\phi(m ; k)$. If the wavelet transform of a function is to be considered only over a finite number of scales

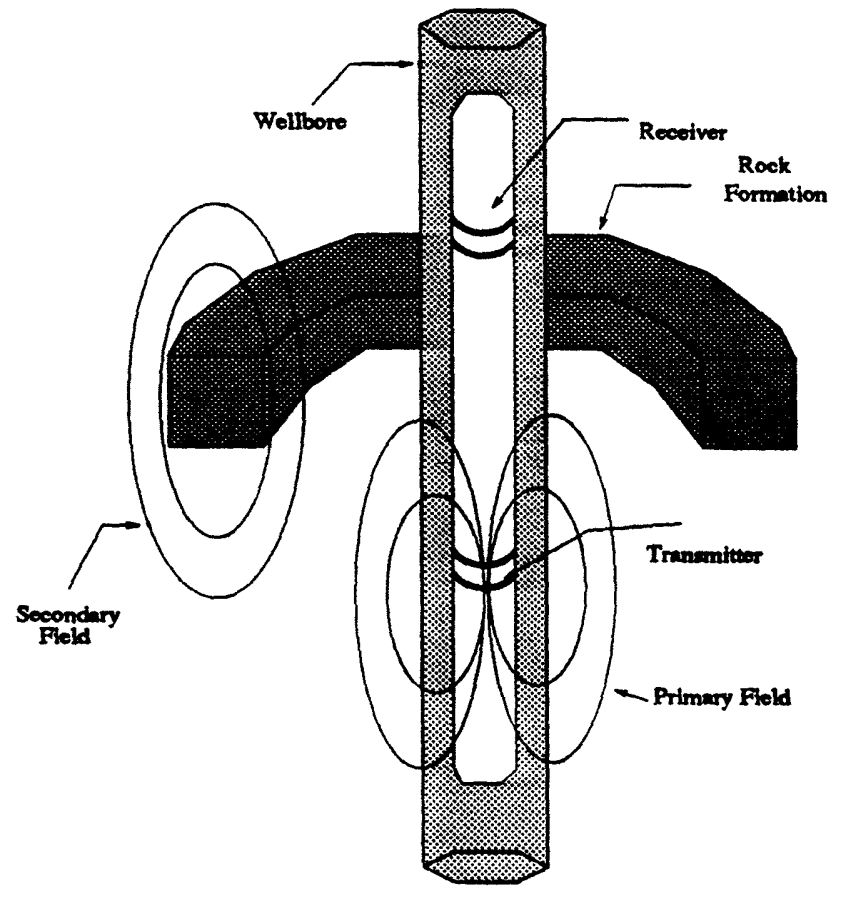

Figure 1: Geometry of Induction Logging

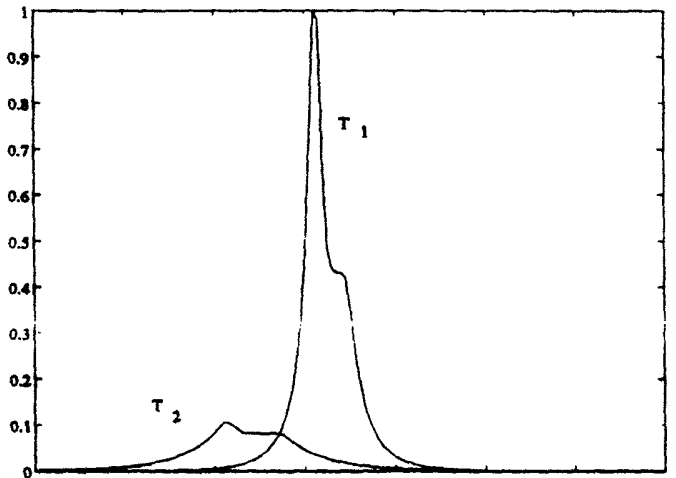

Figure 2: Two Induction Kernels 
them the coarsest scale is denoted $L$ and the finest by $M$. Additionally, in many cases, it is useful to consider the collection of scaling or wavelet coefficients at a particular scale as a single entity. Hence we adopt the notation $\phi(m) \equiv\{\phi(m ; k) \mid k \in \mathbf{Z}\}$ and $f(m) \equiv\{f(m ; k) \mid k \in \mathbf{Z}\}$. When considering the wavelet transform of a function over a finite number of scales: $L \leq m \leq M$, we use the following construct:

$$
\mathcal{W}(f) \equiv \phi=\left[\phi^{T}(M-1) \cdots \phi^{T}(L) f^{T}(L)\right]^{T}
$$

where $\mathcal{W}(f)$ denotes the operation of taking the wavelet transform of the function $f$. In two dimensions we consider only tensor product wavelets [2]. Here, the fundamental grouping of wavelet coefficients for the transform of $T\left(z, z^{\prime}\right)$ is the standard form representation, $\mathcal{W} \mathcal{T}_{i} \mathcal{W}^{*}=\Theta$, as discussed in [2].

Using our notation, Equation 1 may be rewritten as

$$
\gamma=\mathcal{W}(g)=\left[\mathcal{W} \mathcal{T}_{i} \mathcal{W}^{*}\right][\mathcal{W}(f)]=\Theta_{i} \phi
$$

The object of the inversion is to construct and apply $\Theta_{i}^{-1}$ so that we may obtain $\phi$ given the wavelet transform of the function $g(z)$. Then, $f(z)$ is recovered via the inverse wavelet transform.

An example of the structure of $\Theta$ for the conductivity deconvolution problem is given in Figure 3 for the convolution kernel corresponding to $T_{1}(z)$ and using the Haar wavelet for the transform. Following [5], we expect the inverse of $\Theta_{1}$ to be sparse and constructible in a computationally efficient manner. Unlike, [5] however, we propose a stochastic inversion scheme based upon a multiscale a priori probabilistic model for the wavelet transform of $f(z)$.

\section{A STOCHASTIC REGULARIZATION APPROACH}

Two common difficulties associated with inversion problems of the form considered in Equation 1 are illposedness and ill-conditioning of the discretize system. The solution to these issues usually comes in the form of a regularization procedure designed to constrain the original problem so as to make it well-posed and to remove the numerical difficulties associated with poorly conditioned linear systems. In this work, we choose a probabilistically based regularization approach.

\subsection{The Regularization}

The regularization is accomplished in two steps. First, we assume that the measurements are corrupted by an additive, white Gaussian noise process, $n(z)$, so that the in put to the inversion algorithm, $y(z)$, is given by

$$
\begin{aligned}
y_{i}(z) & =g_{i}(z)+n_{i}(z) \\
\Rightarrow \mathcal{W}\left(y_{i}\right) & =\eta_{i}=\Theta_{i} \phi+\nu .
\end{aligned}
$$

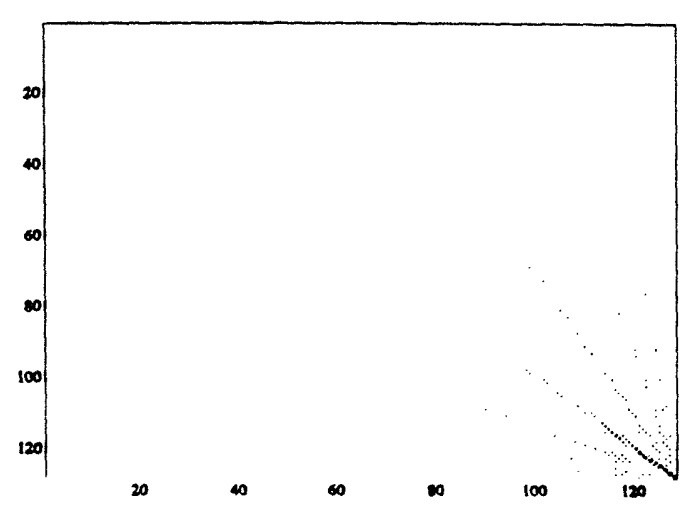

Figure 3: Grayscale Image of $\left|\Theta_{1}\right|$ Using Haar Wavelet

where $\nu=\mathcal{W}(n)$. Second, we construct a prior model for the function $f(z)$. Because we are interested in performing the inversion in the wavelet transform domain, a natural choice for a prior is a multiscale model of the form considered [1]. Here we take the model as

$$
\begin{aligned}
f(m+1) & =H^{*}(m) f(m)+G^{*}(m) \phi(m)(6) \\
\phi(m) & \sim \mathcal{N}(0, R(m)) \\
E\left[\phi(m) \phi(n)^{*}\right] & =R(m) \delta(n-m)
\end{aligned}
$$

where $f(m)$ are the scaling function coefficients of $f(z)$ at scale $m, \phi(m)$ are the corresponding wavelet coefficients, $H(m)$ and $G(m)$ are the wavelet transform analysis operators, and the notation * indicates adjoint. Finally, Equations 7 and 8 indicates that the wavelet coefficient sequence is a zero mean white, Gaussian process with covariance at scale $m$ given by $R(m)$. This particular a priori model defines a stochastic process recursively in the scale parameter $m$ on a lattice of points [1]. More general lattice-type processes can be constructed [1] which may be of use in future work on multiscale inversion techniques.

\subsection{Inversion Algorithms}

Given this multiscale, stochastic regularization we may immediately construct the Maximum a posteriori estimator for the wavelet coefficients of $f(z)$ as [6]

$$
\hat{\phi}_{M A P}=\Lambda_{\phi} \Theta^{T}\left[\Theta \Lambda_{\phi} \Theta^{T}+\Lambda_{\eta}\right]^{-1} \eta
$$

with $\Lambda_{x}=\operatorname{cov}(x)$ and where we have tacitly assumed $i=1$. The form of the gain matrix multiplying $\eta$ for 


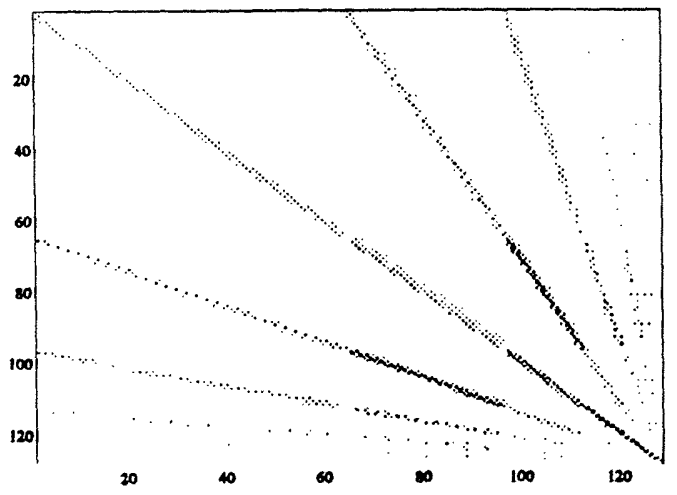

Figure 4: Grayscale Image of Absolute Value of Gain Matrix in Equation 10 Using Haar Wavelet

the kernel $T_{1}(z)$ is given in Figure 4 demonstrating that the stochastic inverse can be a structured and sparse matrix which should be constructible in an efficient manner.

Construction of the inverse according to Equation 9 is not efficient due to the necessity of directly inverting the matrix in brackets. In the presentation accompanying this paper, we discuss inversion algorithms which utilize the system defined by Equations 5 through 8 to synthesize state-space-like models which allow the inversion to proceed in a scale-recursive manner. The model so constructed must meet two criteria. On the one hand, we require some level of fidelity from the model in that it should reproduce the action of the original operator to within acceptable and quantifiable limits. On the other hand, the model must be of sufficiently low complexity so that it lends itself well to computationally efficient inversion algorithms.

Ising these approximate models, we identify three broad classes of inversion procedures which may be considered. First, the models may be both sufficiently accurate and of sufficiently low complexity such that estimates obtained using these models represent the final solution to the inverse problem. Alternatively, we may utilize the approximate models as pre-conditioners for other solution techniques such as successive over relaxation. Finally, it may be possible to improve the estimates generated under out approximate models using an iterative correction scheme built around the multiscale model itself rather than an SOR-type approach.

\section{CONCLUSION and FUTURE WORK}

This paper has presented the framework for a multiscale approach to the solution of one dimensional inverse problems. We have discussed the utility of this approach, demonstrated its potential through the use of a relevant example, and developed the basics of a multiscale stochastic regularization method for solving the problem. In the presentation accompanying this paper, we discuss scale-recursive, computationally efficient reconstruction algorithms derived from statespace-like models constructed to approximate the original operator.

\section{References}

[1] Kenneth C. Chou, S.A. Golden, and Alan S. Willsky. Multiresolution stochastic models, data fusion and wavelet transforms. Technical report, MIT Laboratory for Information and Decision Systems, 1992.

[2] G. Beylkin, R. Coifman, and V. Rokhlin. Fast wavelet transforms and numerical algorithms $I$. Communications on Pure and Applied Mathematics, 44:141-183, 1991.

[3] Darwin Ellis. Well Logging for Earth Scientists. Elsevier, 1987.

[4] J.H. Moran and K.S. Kunz. Basic theory of induction logging and application to the study of two coil sondes. Geophysics, 1962.

[5] B. Alpert, G. Beylkin, R. Coifman, and V. Rokhlin. Wavelets for the fast solution of second-kind integral equations. Technical report, Yale University, 1990.

[6] Alan S. Willsky. Class notes: MIT course 6.433 (recursive estimation). 